\title{
LA PERSPECTIVA DE GÉNERO EN LA EDUCACIÓN Un acercamiento la necesidad de una educación que incluya la perspectiva de género
}

The gender perspective in Education. An approach to the need for an education that includes the gender perspective

Paola Polo Medina

Universidad Femenina del Sagrado Corazón paopmedina@yahoo.es

\section{RESUMEN}

A través de estas páginas, se presentará un breve bosquejo de la perspectiva de género y la necesidad de ser introducida con seriedad en la educación. Es un tema relevante, y asi lo será, mientras las mujeres sigan siendo postergadas de la sociedad en algún grado. Por eso, y ante la realidad de demonización de las luchas por la igualdad de género y del feminismo que las reivindica; este documento intenta suscitar el interés por conocer más de cerca estos temas para asi poder optar con un criterio maduro o dejar de lado con un fundamento razonable. Es decir, invita a evitar que se actúe según el movimiento de las masas sino desde el criterio sensato y propio que da el saber o conocer la expresión de un fenómeno o realidad cualquiera. Este texto, así mismo, invita a la tolerancia desde el conocimiento de la realidad y no en base a cimentar la diferencia fruto de la falta de diálogo abierto y sincero.

Palabras clave: Educación, formación integral, universidad, perspectiva de género, mujer, inclusividad, equidad, feminismo, exclusión, sexismo, dignidad, igualdad, holístico, formación para la vida, cultura de paz.

Fecha de Recepción: 14 de agosto de 2017

Fecha de Aceptación: 11 de octubre de 2017

\section{ABSTRAC}

Through these pages, will be presented a brief outline of the gender perspective and the need to be introduced seriously in education. It is a relevant issue, and it will be so, as long as women continue to be deferred from society to some degree. For this reason, and in view of the reality of the demonization of the struggles for gender equality and feminism that claims it, this document tries to arouse the interest to know more about these issues and thus be able to choose it with a mature criterion or leave it on the side with a reasonable foundation. That is to say, to avoid acting according to the movement of the masses but from the sensible and proper criterion that gives the knowledge or know the expression of a phenomenon or any reality. This text, likewise, invites tolerance from the knowledge of reality and not to cement the difference resulting from the lack of open and sincere dialogue.

Keywords: Education, integral formation, university, perspective of gender, woman, inclusivity, equity, feminism, exclusion, sexism, dignity, equality, holistic, formation for the life, culture and peace. 


\section{INTRODUCCIÓN}

En la realidad peruana, como en muchos países a nivel mundial, el tema de género es una cuestión vigente y con necesidad de abordar con madurez. No obstante, la cuestión o perspectiva de género y el feminismo, que es su voz viva, han de ser bien difundidas, pero para eso, primero deben ser bien comprendidos. Y es que no se puede hablar de una realidad si no se la conoce. De lo contrario, puede terminar por utilizarse el desconocimiento que se tiene de ella para manipularla bajo sus propios intereses.

La realidad descrita en el párrafo anterior es, justamente, la que está detrás de este escrito. Por tanto, lo que motiva a compartir o poner sobre la mesa este tema, es la necesidad de una buena interpretación de la cuestión de género que reivindica el feminismo teórico e incluso el feminismo teológico, en busca de la dignidad de las mujeres (Salto, 2007) y su inclusión, en igualdad, en la sociedad a nivel mundial. Ahora bien, qué mejor que la educación para formar con madurez, acerca de la cuestión de género. Es necesario, y no podemos ser indiferentes, pues constituye un signo importante de nuestro tiempo (Polo, 2015); y es urgente para que las mismas mujeres y la sociedad en general crezca hacia un mundo que ha llegado a su mayoría de edad, como diría Bonhoeffer (citado en Gibellini, 1993).

Aunque es cierto que la educación es un largo proceso que inicia en casa, en la familia, en el barrio y que la educación básica es fundamental para los primeros años de la formación integral, en este escrito vamos a poner el tema de género en relación a la educación superior que nos coloca a un paso de la vida laboral, desde donde nos manifestaremos como constructores de la sociedad de un modo específico. De aquí la convicción de la importancia de que la educación con perspectiva de género esté vigente y sea real en la educación superior: para que no se sigan replicando los estereotipos exclusores que relegan a las mujeres a los márgenes de la sociedad y de la historia.

Pues bien, para introducirnos en esta temática, seguiremos el siguiente recorrido: En la primera parte se desarrollará acerca de las dificultades que afronta la cuestión de género en la sociedad. En un segundo momento se aborda la perspectiva de género propiamente dicha y su importancia para la formación integral de las personas. Luego, y en último lugar, terminaremos con algunas conclusiones para cerrar esta temática.

\section{LA CUESTIÓN DE GÉNERO EN CONTEXTO: LA DEMONIZACIÓN DEL FENÓMENO}

Llama la atención que el mundo haya dado grandes pasos en la modernidad y en la época contemporánea, pero todavía estén vigentes fuertes signos de exclusión acerca de las mujeres. Esta exclusión, que está fundamentada en el sexismo, viene desde el mundo griego y el dualismo filosófico que separa el mundo en dos: buenos - malos, blanco - negro, luz - oscuridad, hombre - mujer, etc. Así mismo, hay que decirque este sexismo se expresa desde dos vertientes que están muy presentes en nuestra sociedad: el patriarcalismo y el androcentrismo. Ambos, respectivamente, consisten en ubicar a las mujeres en los últimos estratos de la pirámide social y considera que el hombre es expresión plena de todo lo humano. ¿Qué significa esto? Que la mujer es una expresión menor de lo humano, como de menos categoría (Johnson, 2003).

No es difícil escuchar frases replicadas en los medios de comunicación tales como que las mujeres son las culpables de que los hombres las agredan o las violen, incluso comentarios que no solo proceden de hombres, sino también de otras mujeres. Tampoco es poco frecuente escuchar hablar que las mujeres en la empresa "X", trabaja por un sueldo menor, por idéntico trabajo realizado por un hombre.

Esto, lamentablemente ha llevado a que las luchas de las mujeres por sus derechos sean vistas como una cuestión sin sentido y que, en ocasiones, las mujeres sean consideradas como manipuladoras sociales.

Otro punto a explorar, es el que la lucha por la cuestión de género ha sido estrecha y únicamente ligada con el homosexualismo y el aborto. No obstante, el feminismo, independientemente de su expresión concreta, es simpley llanamente el reclamo de la dignidad de las mujeres. En este sentido, llamar a las feministas, abortistas o comunistas es una afirmación demasiado rápida que necesita ser repensada y corregida con mucho respeto y tolerancia. Se tendrá que partir por ejemplo, de ver 
la existencia de que no hay un único feminismo, sino una amplia diversidad de expresiones.

En suma, se hace necesario conocer realmente qué es la cuestión de género que reclama el feminismo-en general-y ser críticos con fundamento y veracidad, con coherencia y respeto.

\section{LA PERSPECTIVA DE GÉNERO}

\section{1. ¿Qué es la perspectiva de género?}

Es la categoría de estudio de la teoría feminista. El punto de partida de esta categoría es que la idea de lo femenino y lo masculino es un constructo cultural. En este sentido, la perspectiva de género se opone al sistema social forjado por el sexismo. Así, mientras el sexismo, entre otras cosas, divide a la sociedad por diferencias funcionales entre mujeres y hombres, la categoría de género denuncia esta división como deshumanizadora porque las ubica en un puesto de inferioridad en base a una idea creada por ellos.

Aunque el término género no es nuevo, pues tiene su génesis en el s. XVII gracias a Poulain de la Barre y se ha consolidado a lo largo de los años (Cobo, 1995), es un tema bastante tenso en nuestra realidad local, pues la cuestión de género ha dividido a la sociedad en dos (Rousseau, citado en Cobo, 1995). A lo que apunta el tema de género no es negar las diferencias biológicas entre hombres y mujeres, porque las hay.

Lo que esta visión reclama es la inferioridad y negación con que han sido comprendidas las mujeres a partir de la diferencia biológica. Pero, atención, no por lo biológico, si no por la función que la sociedad sexista le ha asignado a ella, por esa razón: la mujer ha de permanecer en el ámbito doméstico y el hombre en el ámbito público (Millet, citado en Cobo, 1995).

La perspectiva de género en los estudios ha atravesado todos los temas sociales y ha traspasado todos los ámbitos y niveles sociales. Bajo este dato relevante, podríamos preguntarnos, por qué no ha de introducirse en la educación. Sería una gran herramienta para reconocer la realidad de las mujeres con más certeza y podría contribuir a la cultura de paz que el mundo pregona como un anhelo a alcanzar.
Así, si los estudios de género han modificado el pensamiento social y político al introducir la variable sexo como nueva categoría de análisis" (Ibídem, p. 65), puede ser capaz de transformar la educación hacia una verdadera formación integral y humanista o humanizadora. De lo contrario, la educación seguirá contribuyendo a la división del mundo, al subdesarrollo de los países y al reforzamiento de una sociedad desigual. En este sentido, me atrevo a decir, que la educación tiene el deber moral y la responsabilidad de - preparar para la vida y contribuir al desarrollo de los pueblos- por lo que ha de incluir en sus programas la perspectiva de género.

\subsection{La perspectiva de género como política para la inclusividad \\ Es obvio que la mayor implicancia dela urgencia} por incluir la perspectiva de género en la educación es la vida en igualdad entre hombres y mujeres, así como la dignidad de ellas. Hay implicancias subcontenidas que permiten la posibilidad de acceder a una vida más equitativa. Así, por ejemplo, podemos hablar de la inclusión demográfica. Es decir, permitir el acceso a la universidad de hombres y mujeres, sin discriminación de carreras y oportunidades becarias.

También hay que pensar en la inclusión de la perspectiva de género, en la práctica, tanto en el ámbito administrativo como en la práctica docente en las aulas. Practicar el cuestionamiento crítico antes las desigualdades sociales, la falta de oportunidades con equidad para las mujeres, las visiones sexistas, la violencia de género, etc. (Palomar, 2004).

Pero la perspectiva de género no es un tema de moda que toda mujer debe asumir; es decir, no es una cuestión que implica solo a las mujeres que desean ser reconocidas en su dignidad. El tema de género es mucho más amplio y abarca la responsabilidad de cada persona, de cada gobierno y de cada institución internacional que tiene en sus manos organizar la sociedad.

Dehecho, unodelosorganismosinternacionales como la ONU, dice que es necesario promover los objetivos de igualdad, desarrollo y paz para todas las mujeres del mundo, en interés de toda la humanidad". De hecho, la ONU ha creado la ONU Mujeres, cuya función es: 
Dar apoyo a las entidades intergubernamentales como la Comisión de la Condición Jurídica y Social de la Mujer en su formulación de políticas y estándares y normas mundiales, dar asistencia a los Estados Miembros para implementar esos estándares, dando cuando sea necesario el apoyo técnico y financiero adecuado para ayudar a los países que lo soliciten, así como para establecer alianzas eficaces con la sociedad civil; y hacer que el sistema de la ONU rinda cuentas de sus compromisos en materia de igualdad de género, incluyendo el monitoreo continuo de los progresos dentro del sistema. (ONU).

Otra de las entidades internacionales que ha influido en la acción social por la inclusividad de géneroenlosámbitosnacionaleshasidolaUNESCO. Según esta organización queda claro que la cuestión de género y su lucha no es solo cuestión de mujeres. Afirma que "igualdad de género significa igualdad de derechos, responsabilidad y oportunidades para mujeres y hombres y para niñas y niños". Esto no es otra cosa la apelación a la responsabilidad de todos y a las políticas gubernamentales e incluso educacionales, pues hablar de igualdad de género es hablar-dice- de derechos humanos.

Influida por las organizaciones internacionales, y afiliando a los acuerdos, en el Perú se asume la cuestión de género en la política social y en la educación. Así, en Perú el Ministerio de la Mujer (MIMDES), por Decreto Legislativo $\mathrm{N}^{\circ}$ 866, promueve en el ámbito local, la equidad de género y la igualdad de oportunidades para las mujeres.

Así mismo, se ha establecido la formulación de políticas públicas como el Plan de Igualdad de Oportunidades para Mujeres y Varones; que se ha ido renovando con el pasar de los años y -lo mejores que implicó a más ministerios (Plan Nacional de Igualdad de Género 2012 - 2017, el PLANING).

\subsection{La perspectiva de género en la educación}

Aunque hay diferencias de oportunidades y de igualdad en la cuestión de género a nivel nacional, la mayor señal de inequidad se da en el ámbito rural (PLANING, 2012 - 2017), a pesar de que ha habido cambios positivos para el acceso de las mujeres a la educación. De este modo, vemos que, aunque la brecha se acorta, persiste en desmedro de las mujeres. Así, en 2011, las mujeres del área rural acceden a la matrícula para secundaria en un $57.8 \%$, a diferencia de los hombres que están en un $66 \%$. Del mismo modo, las mujeres rurales siguen teniendo el índice más alto de analfabetismo 14.4 $\%$ (Ibídem).

Aparte de estos datos, que reflejan el menor acceso de las mujeres a la educación, esto supone algo de mucha más envergadura: las limitaciones de las mujeres para el sector laboral, que refuerzan la idea de que la mujer debe estar en la cocina, la poca participación en la política y las decisiones gubernamentales, su aporte a la economía, etc. en este sentido, hay que decir que mientras las mujeres sigan teniendo menos acceso a la educación, seguirán siendo relegadas. Por tanto, la educación debe ser el instrumento clave para la erradicación de la desigualdad de las mujeres, en particular, y las desigualdades sociales, en general.

\section{a. La Ley General de Educación 28044}

Además de establecer la educación como derecho fundamental, la Ley General de Educación afirma que ella contribuye a la formación integral de las personas (Art. 2 y 3). Esto significa que, si las mujeres no tienen acceso a la educación o no con el mismo nivel de acceso a ella, este desarrollo no podrán alcanzarlo muchas mujeres.

Del mismo modo, se plantea como uno de los principios de la educación (Artículo 8, inciso b - c), la equidad y la inclusión. Garantiza la igualdad de oportunidades sin ningún tipo de distinción por raza, etnia, sexo, etc. Es más, uno de los fines de la educación, según el artículo 9, inciso b; es formar sociedades desarrolladas desde la inclusividad de la diversidad, con la participación de todos en este propósito. No obstante, si la mujer está lejos de los hilos que atan la historia, nunca podrá participar en el desarrollo de la sociedad.

\section{b. Ley Universitaria 30220 de Perú}

Aunque no expresamente, la Ley Universitaria, parece incluir la cuestión de género en sus líneas, al considerar como parte de sus principios la democracia institucional (5.6), con lo cual habría que pensar en la participación también de las mujeres en puestos altos, y en acceso de ellas a una carrera universitaria con las mismas facilidades que los hombres. 
En el inciso 5.8, habla de pluralismo, tolerancia, diálogo intercultural e inclusión; que, aunque es sabido que no es solo la inclusión de las mujeres, sino también de las personas con alguna discapacidad. Esto se refuerza con el inciso 5.10, que dice que otro principio es la afirmación de la vida y la dignidad. Aunque suene repetitivo, hay que afirmar entonces, que no hay dignidad en la sociedad si no la hay también para las mujeres.

Estos principios mencionados, y los otros, servirán de base para establecer las funciones de la universidad; por tanto, de aquí saldrá su hacer. En este sentido, a la universidad le compete participar de la formación integral e inclusiva que involucre también el bien de las mujeres; y esto será más fácil si asume las políticas de la igualdad de género y su perspectiva como línea transversal para un mejor desarrollo humanista e integral.

\subsection{Justificación de la perspectiva de género en la educación universitaria}

Ahora bien, ¿qué justifica que todo lo dicho anteriormente deba ser tomado en serio y deba pasar del solo compromiso internacional a ser una praxis nacional de influencia humanista y humanizadora de la vida en la realidad contemporánea?

Hay que empezar diciendo que hay jóvenes -incluso pertenecientes a universidades- que sufren violencia de género y no saben que la padecen, hay quienes no conocen sus derechos y no saben lo que es acoso sexual callejero o al menos lo malentienden (Vélez, s/f).

Según estudios hechos por la universidad San Martín de Porres, el 65 \% de mujeres de estudiantes universitarias son agredidas por sus parejas, el $47.8 \%$ son agredidas en el último año. Esto trae como consecuencia, según el mismo estudio, un menor rendimiento académico, mayor deseo de deserción, más regaños de docentes y colegas y más preocupaciones; en las mujeres que en los hombres. Para ser más precisos, las mujeres pierden 20.4 días de clase por la violencia de género. Otro dato importante es el tipo de violencia que se padece, que no solo es la física; sino también la psicológica, la sexual y hasta la económica (USMP, s/f).

Una de las razones de la educación es la formación para la vida. Es decir, prepararte para la vida laboral, ente otras cosas. Sin embargo, una vez acabada la universidad, aún hoy las mujeres deben luchar por un sueldo y un puesto justo, cosa que en muchos sectores es más fácil para los hombres y deben pelear además por un sueldo igual y digno por el mismo trabajo que hacen ellos.

Pero, los datos del INEI reflejan que aún queda un largo recorrido para que la equidad de género llegue a estos estratos. Así, por ejemplo, la desigualdad de género en cuanto a la participación de la fuerza laboral se expresa en un $63.4 \%$ para las mujeres y un $82,5 \%$ para los hombres (INEI, 2016).

\section{CONCLUSIONES}

Para dar final a esta temática, sobre la cuestión de género y la educación, finalizo con algunas ideas a modo de conclusión.

1. La cuestión de género es un tema no reciente, pero aún vigente, que necesita ser abordado con madurez social y de forma integral. Siendo una categoría de análisis social, ha de traspasar todas las disciplinas también, la educación. Y debe dejarse de lado los estereotipos creados que terminan por demonizar un fenómeno social que aporta al proceso humanizador de la sociedad en favor de una sociedad de paz y justicia.

2. La perspectiva de género ha sido acogida por medio de las instituciones internacionales relacionadas con los intereses de la defensa de los derechos humanos, pero, en la práctica, aunque se han visto avances y las mujeres han ido ganando terreno por un puesto digno, aún queda camino por recorrer.

3. Laeducación, desdela normatividad, ha acogido también los lineamientos de la igualdad de género, pero no parece ser un tema con mucha presencia y que genere cambios sustanciales a nivel general- en favor de la dignidad de las mujeres. Así, sería bueno proponer políticas institucionales que conduzcan a la buena convivencia y a contribuir a una cultura de paz, a través de la reinvidicación de la dignidad de las mujeres.

4. La educación universitaria tiene, en su poder, la facultad de contribuir al crecimiento social, 
político, económico y cultural. Y es que la universidad es un campo propicio para formar, desde la ética y demás disciplinas transversales. A través de ella, los hombres y mujeres se perfilarán como sujetos activos para el mundo futuro. De ellos depende qué mundo quieren construir: si de inclusión o exclusión, si un mundo unido a pesar de las diferencias o un mundo dividido desde las mismas diferencias.

5. Finalmente, para concluir, hay que decir que ningún país alcanzará el objetivo de alcanzar la cultura de paz, la inclusividad de todo tipo, la formación integral y humanista ni la formación para la vida si no se realiza la real inserción de las mujeres en todos los ámbitos, como coconstructoras eficaces de la historia y la cultura.

\section{REFERENCIAS}

Cobo, R. (1995). "Género". En Amorós, C. (Ed.), 10 palabras clave sobre Mujer (pp. 55 - 83). Estella: Verbo Divino.

Gibellini, R. (1993). La teología del siglo XX. Santander: Sal Terrae.

Johnson, E. A. (2003). La cristología, hoy. Olas de renovación en el acceso a Jesús ( $2^{\text {a }}$ Edición). Santander: Sal Terrae.

INEI (2016). Perú: Brechas de Género 2016. Avances hacia la igualdad de mujeres y hombres. Recuperado de: $\quad$ http://www.inei.gob.pe/media/ MenuRecursivo/publicaciones_digitales/Est/ Lib1388/Libro.pdf

Minedu (2003). Ley General de Educación. Recuperado de http://www.minedu.gob.pe/ p/ley_general_de_educacion_28044.pdf

Minedu (2014). Ley Universitaria $N^{\circ} 30220$. Recuperado de http://www.minedu.gob.pe/ reforma-universitaria/pdf/ley_universitaria. pdf

Ministerio de la mujer y poblaciones vulnerables (2012). Plan Nacional de Igualdad de género 2012 - 2017. Recuperado de https://www.mimp. gob.pe/files/planes/planig_2012_2017.pdf
Organización de las Naciones Unidas (ONU). Recuperado de http://www.un.org/ youthenvoy/es/2013/07/onu-mujeresentidad-de-las-naciones-unidas-para-laigualdad-de-genero-y-el-empoderamientode-las-mujeres/ también ver http://onu.org. $\mathrm{pe} /$ temas/derechos-de-la-mujer-y-equidad-degenero/

Palomar, C. (2004). La politica de género en la educación superior. México: La teoría.

Polo, P. (2015). Las mujeres en la Iglesia, de discípulas a olvidadas y a sujetos de liberación. Phainomenon. 15 (1), pp. $79-92$.

Salto, A. (2007). La dignidad humana. Dignidad de la mujer. Madrid: PPC.

Unesco. Igualdad de género. Recuperado de http:// es.unesco.org/creativity/sites/creativity/files/ digital-library/cdis/Iguldad\%20de\%20genero. pdf

Unesco. Igualdad de género. Recuperado de http:// www.unesco.org/new/es/havana/ areas-ofaction/ igualdad-de-genero/

Universidad SanMartín de Porres (s/f). Estudio "La violencia contra las mujeres en las universidades peruanas". Recuperado de http://www. mujereslibresde violencia.usmp.edu.pe/ estudio-violencia-contra-las-mujeres-en-lasuniversidades-peruanas/

Vélez, G. (s/f). La incorporación de la perspectiva de género en la universidad autónoma del Estado de México. Logros, retosy obstaculización. Recuperado de https://www.google.com.pe/search?q=La + incorporaci $\% \mathrm{C} 3 \% \mathrm{~B} 3 \mathrm{n}+\mathrm{de}+\mathrm{la}+$ perspectiva + de $+\mathrm{g} \% \mathrm{C} 3 \% \mathrm{~A} 9$ nero+en+la + universidad + aut $\% \mathrm{C} 3 \% \mathrm{~B} 3$ noma + del+Estado+de+M $\% \mathrm{C} 3 \% \mathrm{~A} 9$ xico. + Logros $\% 2 \mathrm{C}+$ retos $+\mathrm{y}+$ obstaculizaci $\% \mathrm{C}$ 3\%B3n\&oq=La+incorporaci\%C3\%B3n+de+1 $\mathrm{a}+$ perspectiva $+\mathrm{de}+\mathrm{g} \% \mathrm{C} 3 \% \mathrm{~A} 9$ nero + en $+\mathrm{la}+$ un iversidad+aut $\%$ C3\%B3noma+del+Estado+de $+\mathrm{M} \% \mathrm{C} 3 \% \mathrm{~A} 9 \mathrm{xico} .+$ Logros $\% 2 \mathrm{C}+$ retos $+\mathrm{y}+$ obs taculizaci $\% \mathrm{C} 3 \% \mathrm{~B} 3 \mathrm{n} \&$ aqs $=$ chrome. .6 